\title{
Editorial
}

\section{Nanostructured Materials for Electronics and Photonics}

\author{
R. Bayati, ${ }^{1}$ Y. K. Mishra, ${ }^{2}$ N. Naseri, ${ }^{3}$ and S. Nori $^{4}$ \\ ${ }^{1}$ Intel Corporation, IMO-SC, Santa Clara, CA 95054, USA \\ ${ }^{2}$ Functional Nanomaterials, Institute for Materials Science, University of Kiel, 24143 Kiel, Germany \\ ${ }^{3}$ Department of Physics, Sharif University of Technology, Tehran 11155-9161, Iran \\ ${ }^{4}$ Department of Materials Science and Engineering, North Carolina State University, Raleigh, NC 27695, USA
}

Correspondence should be addressed to R. Bayati; mbayati@ncsu.edu

Received 23 February 2014; Accepted 23 February 2014; Published 2 April 2014

Copyright (C) 2014 R. Bayati et al. This is an open access article distributed under the Creative Commons Attribution License, which permits unrestricted use, distribution, and reproduction in any medium, provided the original work is properly cited.

\begin{abstract}
This special issue addresses both experimental and theoretical research works in the areas of nanoscale electronic, optoelectronic, and magneto-optical materials. It aims to facilitate the dissemination of interdisciplinary research results in the interrelated and rapidly converging fields of nanoelectronics and photonics. The main focus of this issue is placed on enhancing the performance of electronic devices using nanoscience and nanotechnology. The following aspects were considered as particular interests for this special issue:
\end{abstract}

(i) electronic, optical, and magneto-optical properties of semiconductor oxides and inorganic, organic, and hybrid nanostructures,

(ii) electronic applications of superlattices, quantum structures, and other nanostructures,

(iii) optoelectronic and photonic applications of novel materials and nanostructures,

(iv) oxide based nanoelectronic circuits and device integration,

(v) molecular electronics,

(vi) nanobioelectronics,

(vii) nanofabrication, processing, and characterization techniques,

(viii) thin film epitaxy of oxides and nitrides.

In the paper entitled "Nonlinear optical properties of a MMA-silica nanohybrid material doped with Rhodamine 6G," a simple and inexpensive optically nonlinear nanohybrid material is developed, based on a TEOS:MMA nanoporous matrix. The potential applications for this material include lasers, photochromic coatings, and photoluminescent materials for organic devices and biomedical imaging. Nanoparticles of the hybrid material doped with an organic dye show optical nonlinear response due to the dopant. Optical response control depends on the thin film thickness or on the amount of dopant. However, the absorbance and PL spectra taken on the TEOS:MMA hybrid matrix after doping was essentially unaltered, since the organic dopants are transparent over the visible spectral region.

In the paper entitled "Influences of In $\mathrm{GaP}$ conical frustum nanostructures on the characteristics of GaAs solar cells," InGaP conical frustum nanostructures are fabricated on top of the GaAs solar cells and the optical and photovoltaic characteristics of the conical frustum nanostructured solar cells are studied. The most striking finding in this research work is that an increase of $15.2 \%$ in the conversion efficiency is achieved at the optimal geometry with a $100 \mathrm{~nm}$ height and $300 \mathrm{~nm}$ top diameter of the InGaP conical frustum nanostructure. The improvements are attributed to the gradual change in the refractive index, resulting in the Fresnel reflection suppression and light scattering effect by the InGaP conical frustum nanostructures.

The fabrication of InAs quantum dots (QDs) on InGaAs/GaAs is addressed in the paper entitled "InGaAs quantum dots on cross-hatch patterns as a host for diluted magnetic semiconductor medium." In this research publication, the authors have demonstrated clearly how to grow InAs 
QDs on InGaAs/GaAs using molecular beam epitaxy (MBE). The results are important due to the fact that the lattice-mismatched heteroepitaxy can be used to form laterally aligned, high-density semiconducting host in a single growth run without any use of lithography or etching. Lattice-mismatched epitaxy results in the formation of quantum dots (QDs), cross-hatch patterns (CHPs), stripes, and smooth films in decreasing order of misfit. For low-misfit InGaAs/GaAs, the dislocation lines making up the CHPs are widely spaced, resulting in stripes or cross-hatch patterns. For intermediate-misfit InGaAs/GaAs, surface CHPs are characterized by narrowly spaced dislocation lines along the two orthogonal directions with a different line density. The unidirectionally aligned QDs result in QD chains with electronic and optical coupling between dots while the bidirectionally aligned ones result in QD matrices. With appropriate alloying of a transition element and careful control of average dislocation spacings, these QD matrices can exhibit spin coupling and may serve as a high-density DMS medium/read heads. Such magnetic regions have the ultimate capability to store one bit of information each.

In the paper entitled "Hydrogenic-donor impurity binding energy dependence of the electric field in $G a A s / A l_{x} G a_{1-x} A s$ quantum rings," authors report a variational method with two-parameter trial wave function and the effective mass approximation for calculation of the binding energy of a donor impurity in $\mathrm{GaAs} / \mathrm{Al}_{x} \mathrm{Ga}_{1-x} \mathrm{As}$ cylindrical quantum ring (QR) subjected to an external field. The authors have clarified that donor impurity binding energy is highly dependent on the QR structure parameters (radial thickness and height), impurity position, and external electric field. Based on their accurate investigations, the binding energy increases inchmeal as the QR parameters (radial thickness and height) decrease until a maximum value for a central impurity and then begins to drop quickly. The applied electric field can significantly modify the spread of electronic wave function in the QR and shift electronic wave function from the donor position and then leads to binding energy changes. In addition, they demonstrated the results for the binding energies of a hydrogenic donor impurity as functions of the impurity position and applied electric field.

In the research article entitled "Light output enhancement of In GaN/GaN light-emitting diodes with contrasting indium tin-oxide nanopatterned structures," various nanopatterns on the transparent conducting indium tin-oxide (ITO) layer to enhance the light extraction efficiency of the InGaN/GaN light-emitting diodes (LEDs) are fabricated and a structureproperty correlation is established. Triangular, square, and circular nanohole patterns with the square and hexagonal lattices are fabricated on the ITO layer by an electron beam lithography and inductively coupled plasma dry etching processes. They found the circular hole pattern with a hexagonal geometry as the most effective among the studied structures. Light output intensity measurements resulted in that the circular hole nanopatterned ITO LEDs with a hexagonal lattice show up to $35.6 \%$ enhancement of output intensity compared to the sample without nanopatterns.
Subsequently, the paper entitled "Low-temperature rapid fabrication of $\mathrm{ZnO}$ nanowire $U V$ sensor array by laserinduced local hydrothermal growth" focuses on $\mathrm{ZnO}$ nanowire based UV sensor synthesized by laser-induced hydrothermal growth of $\mathrm{ZnO}$ nanowire. In this regard, it is shown that, by inducing a localized temperature rise using focused laser, $\mathrm{ZnO}$ nanowire array at $\sim 15 \mu \mathrm{m}$ size that consists of individual nanowires with $\sim 8 \mu \mathrm{m}$ length and $200 \sim 400 \mathrm{~nm}$ diameter can be fabricated on gold electrode within $30 \mathrm{~min}$ at the desired position. The laser-induced growth process is consecutively applied on two different points to bridge the micron gap between the electrodes. The resultant photoconductive ZnO NW interconnections display $2 \sim 3$ orders increase in the current upon the UV exposure at a fixed voltage bias. It is also confirmed that the amount of photocurrent can be easily adjusted by changing the number of $\mathrm{ZnO} \mathrm{NW}$ array junctions. The device exhibits clear response to the repeated UV illumination, suggesting that this process can be usefully applied for the facile fabrication of low-cost UV sensor array.

In the paper entitled "Simulation of an improved design for n-electrode with holes for thin-GaN light-emitting diodes," a novel design for $n$-electrode with holes to be applied in thinGaN light-emitting diodes (LEDs) is discussed. Based on their deep investigations on the influence of the n-electrode with holes on the thermal and electrical characteristics of a thin-GaN LED chip, the variations in current density and temperature distributions in the active layer of n-electrodes both with and without holes are very tiny. The percentages of light output from these holes are $29.8 \%$ and $38.5 \%$ for cases with $5 \mu \mathrm{m}$ holes and $10 \mu \mathrm{m}$ holes, respectively; the side length of the n-electrode (L) is $200 \mu \mathrm{m}$. Furthermore, the percentage increases with the size of the n-electrode. They demonstrated that the light output can be increased 2.45 times using the nelectrode with holes design. The wall-plug efficiency (WPE) can also be improved from $2.3 \%$ to $5.7 \%$ while the most appropriate n-electrode and hole sizes are determined by WPE analysis.

In the last paper entitled "Nanocrystallization of coarse primary phases in Al-and Mg-based alloys induced by HCPEB treatment," the authors report a phenomenon associated with high-current pulsed electron beam (HCPEB) treatment: surface nanocrystallization of coarse primary phase in hypereutectic Al17.5Si and quasicrystal alloys after multiple pulses of HCPEB irradiation. They stated that the HCPEB treatment induces superfast heating and diffusion of alloying elements and heterogeneous nucleation in a melting solution, followed by rapid solidification and cooling of the material surfaces. Consequently, nanostructured surface layers can be achieved easily. Nano-Si phase and nanoquasicrystal phase formation on the modified surface layer of hypereutectic Al17.5Si alloy and quasicrystal alloy $\left(\mathrm{Mg}_{37} \mathrm{Zn}_{60} \mathrm{Y}_{3}\right)$ show a potential for surface nanocrystallization of materials with enhanced properties by HCPEB treatment.

R. Bayati

Y. K. Mishra

N. Naseri

S. Nori 

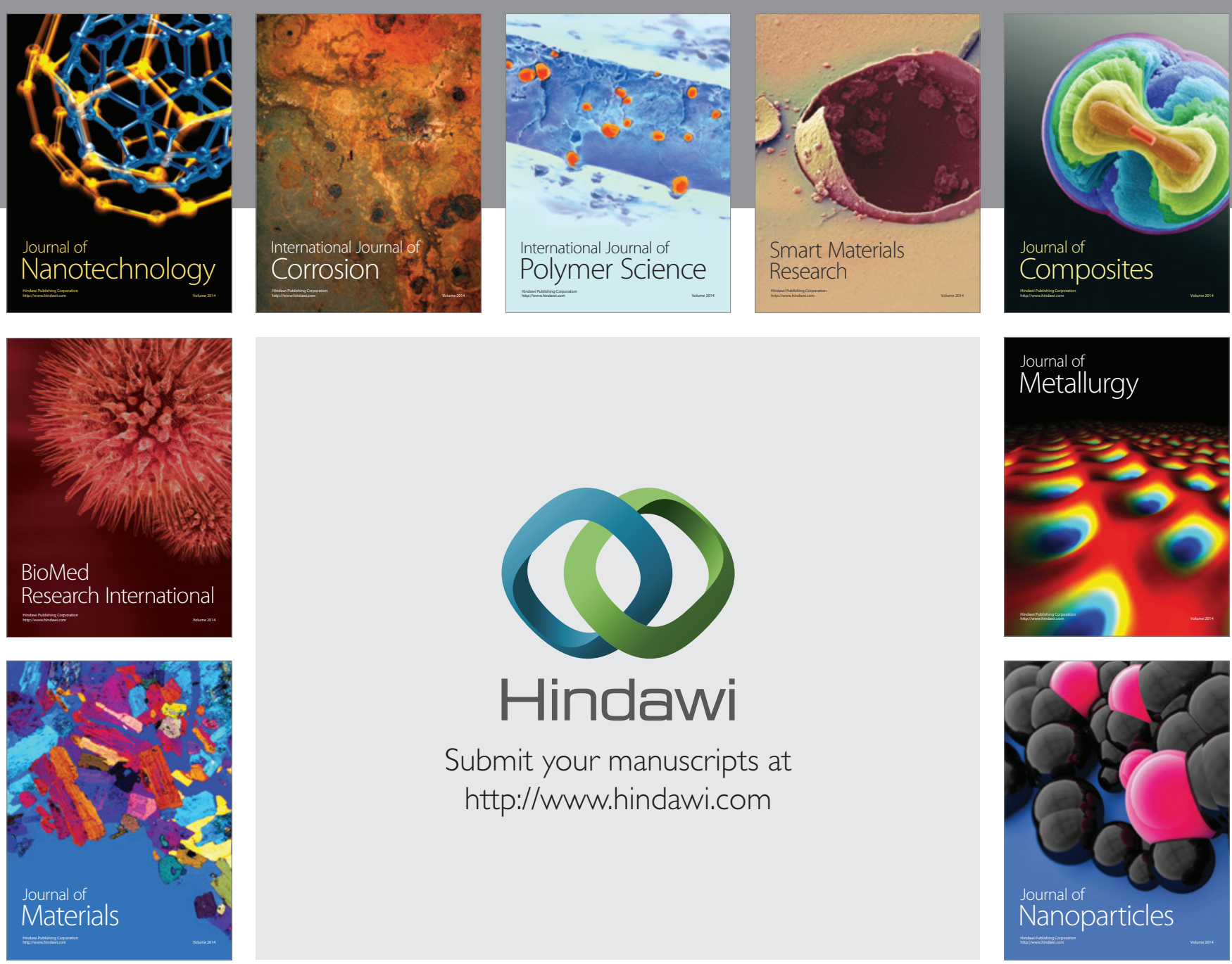

Submit your manuscripts at http://www.hindawi.com
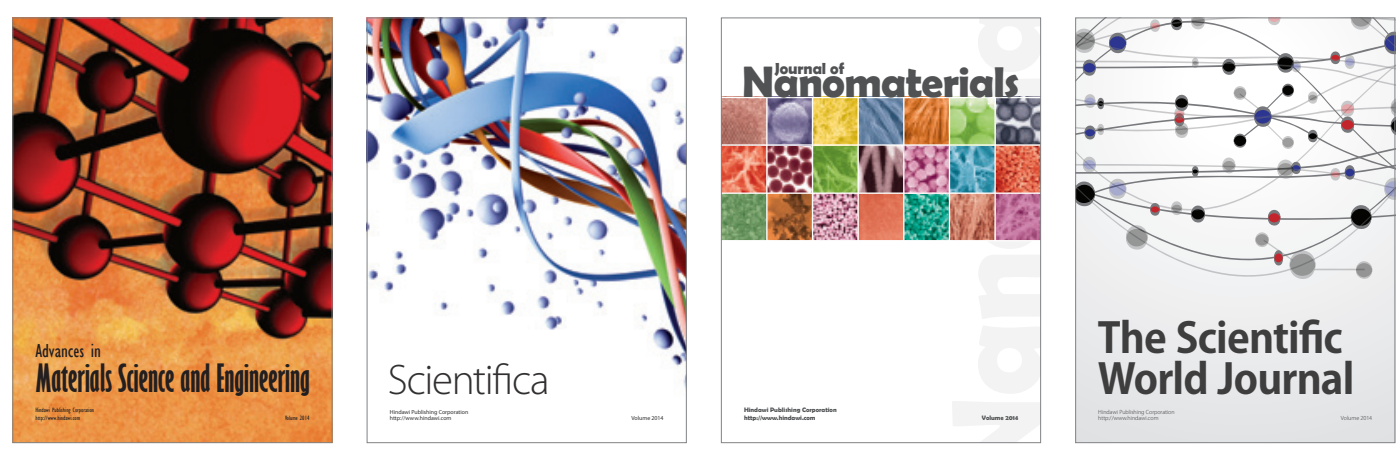

\section{The Scientific World Journal}
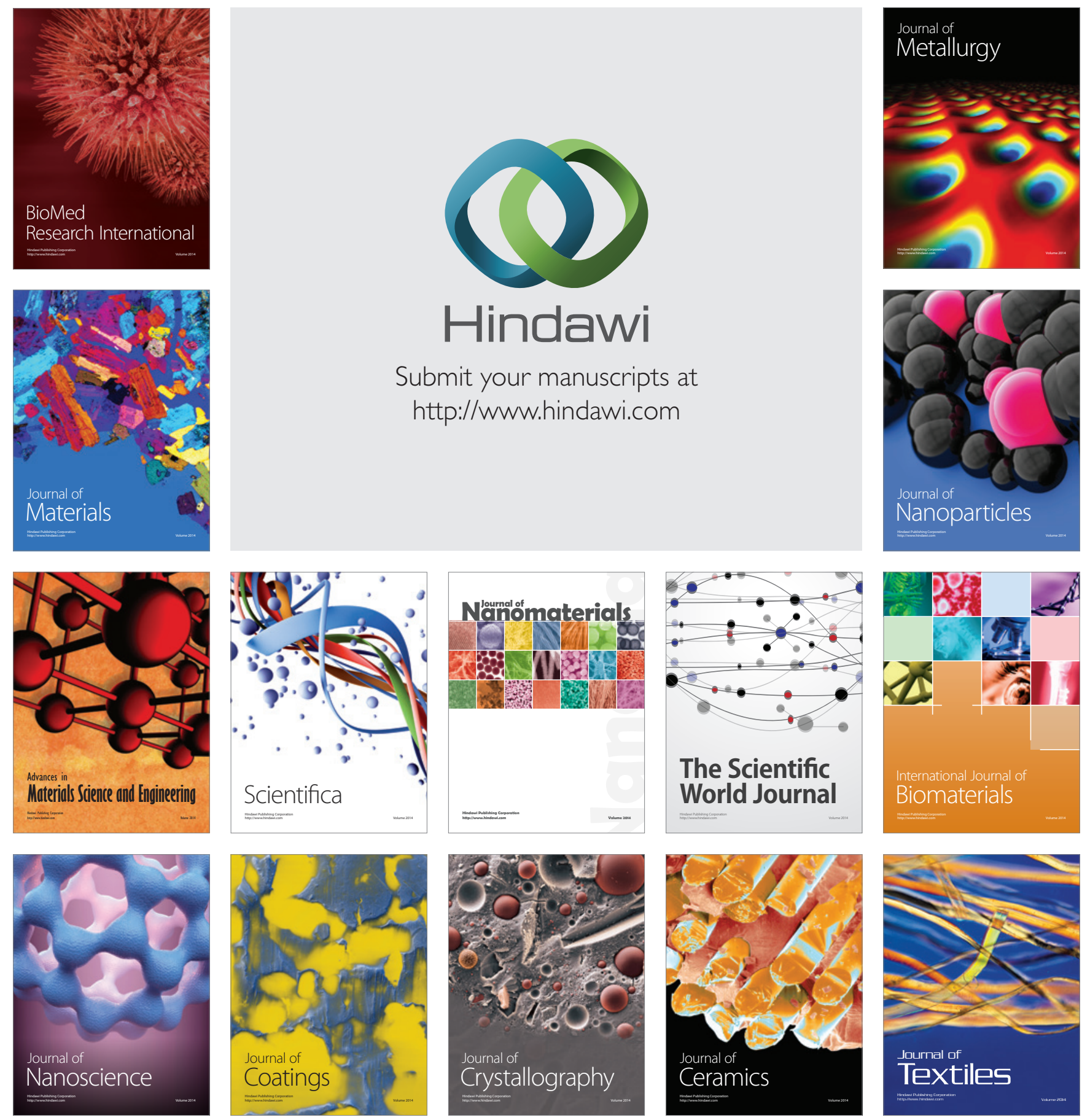